\title{
Influence of prion variant and yeast strain variation on prion-molecular chaperone requirements
}

\author{
Justin K. Hines, ${ }^{\dagger}$ Takashi Higurashi, ${ }^{\ddagger}$ Mathangi Srinivasan and Elizabeth A. Craig* \\ Department of Biochemistry; University of Wisconsin-Madison; Madison, WI USA

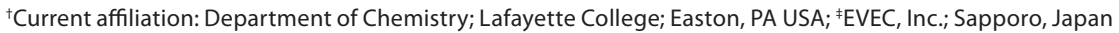

Key words: Hsp40, Ssa, heat-shock, protein misfolding, cell stress, Hsp104, PIN, saccharomyces, neurodegenerative disease

Abbreviations: 5FOA, 5-fluoroorotic acid; SDS-PAGE, sodium dodecyl sulfate polyacrylamide gel electrophoresis; SDDAGE, semi-denaturing detergent agarose gel electrophoresis

Prions of budding yeast serve as a tractable model of amyloid behavior. Here we address the issue of the effect of yeast strain variation on prion stability, focusing also on the effect of amyloid conformation and the involvement of the cochaperone Sis1, an essential J-protein partner of Hsp70. We found, despite an initial report to the contrary, that yeast strain background has little effect on the requirement for particular Sis1 domains for stable propagation of the prion $\left[R \mathrm{RQ}^{+}\right]$, if the level of Sis1 expression is controlled. On the other hand, some variation in prion behavior was observed between yeast strains, in particular, the stability of certain $\left[\mathrm{PSI}^{+}\right]$variants. Future examination of such yeast strain-specific phenomena may provide useful insights into the basis of prion/chaperone dynamics.

\section{Introduction}

Numerous neurodegenerative diseases are associated with the misfolding of a protein into a fibrous, $\beta$-sheet rich form termed amyloid. ${ }^{1}$ Yeast prions, proteins which form heritable amyloid aggregates, have been useful models of amyloid behavior due largely to the tractability of Saccharomyces cerevisiae as a model organism. Two well-studied yeast prions, $\left[P S I^{+}\right]$and $\left[R N Q^{+}\right]$ (also called $\left[P I N^{+}\right]$), are formed by amyloid aggregates of the yeast proteins Sup35 and Rnq1, respectively. ${ }^{2-5}$ Sup35 is a translation termination factor, whereas the cellular function of Rnq1 is unknown. ${ }^{6}$ Within the context of a yeast cell, a critical aspect of prion propagation is the remodeling of amyloid aggregates such that they create heritable seeds, which can be passed on to daughter cells. ${ }^{7}$ Molecular chaperone proteins are critical to this process. ${ }^{7.8}$ Both $\left[P S I^{+}\right]$and $\left[R N Q^{+}\right]$, as well as several less-studied prions, require a specific Hsp70 system, including co-chaperone J-protein and nucleotide exchange factors, working in conjunction with the disaggregase Hsp104..$^{8-11}$ Although the Hsp70 Ssa, involved in prion fragmentation, has many J-protein partners, ${ }^{12,13}$ the J-protein Sis1 is specifically required in yeast prion fragmentation. ${ }^{10,14-17}$

J-proteins, including Sis1, exhibit a modular gene architecture commonly consisting of an N-terminal J-domain, critical for stimulation of their partner Hsp70's ATPase activity, and
C-terminal peptide binding domains (Fig. 1A). ${ }^{12}$ Medial connecting regions are characterized by amino acid abundance, being either glycine/phenylalanine rich (G/F region) or glycine/ methionine rich ( $\mathrm{G} / \mathrm{M}$ region). Cells expressing Sis1 lacking only the G/F region (Sis1- $\Delta \mathrm{G} / \mathrm{F}$, Fig. $1 \mathrm{~A})$ rapidly lose $\left[R N Q^{+}\right]$, indicating an important role for this domain in prion maintenance. ${ }^{10}$ Whether additional domains are required for $\left[R_{N} Q^{+}\right]$propagation is less clear. However, recently a system has been developed to allow more quantitative analysis of Sis1 function in prion maintenance. ${ }^{18}$ Despite being an essential protein, Sis1 expression can be reduced dramatically without serious consequence to yeast cell growth. ${ }^{18}$ Serendipitously, higher levels of Sis1 are required for prion maintenance, permitting repression of Sis1 synthesis to a level that allows cell growth but not prion propagation. This method has been exploited to explore the requirement for Sis1 by analyzing the kinetics of prion loss. ${ }^{14-16,18}$ For 3 prions tested, $\left[R N Q^{+}\right]$, [URE3] and $\left[S W I^{+}\right]$, curing occurs rapidly; cell populations are prion-free $15-25$ generations after the initiation of Sis1 repression by the addition of the tetracycline analog doxycycline. ${ }^{14,16,18}$ In contrast, $\left[\mathrm{PSI}^{+}\right]$curing by this method occurs markedly slower, requiring $>50$ generations for complete curing, highlighting the variation amongst prions in the stringency of the requirement for Sis1 activity for their propagation. ${ }^{14}$

Despite the fundamental requirement for Sis1 and other chaperone components for prion propagation, the behavior of a 


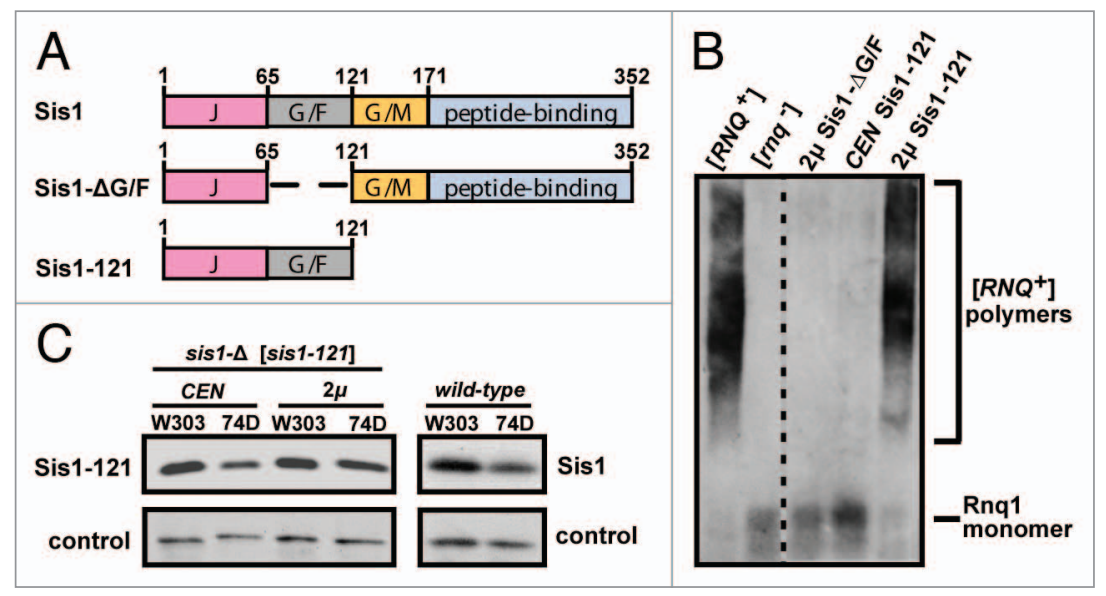

Figure 1. Maintenance of $\left[R N Q^{+}\right]$by Sis1 expression constructs. (A) Gene structure diagrams of Sis1 expression constructs. Gene regions are denoted using the following notation: J, J-domain; $G / F$, glycine/phenylalanine-rich region; $G / M$, glycine/methioninerich region; peptide-binding, C-terminal peptide-binding domains I and II. Dashed line indicates a region has been deleted. (B) Plasmid shuffling experiments to test the domain requirements of Sis1 by $\left[R N Q^{+}\right]$. Plasmids expressing Sis1 constructs illustrated in (A). Detergent resistant Rnq1 aggregates indicative of the presence of $\left[R N Q^{+}\right]$were visualized by SDDAGE followed by immunoblot analysis. Control wild-type $\left[R N Q^{+}\right]$and $[r n q]$ cells were included for comparison. Dotted lines separate lanes taken from different parts of the same gel. (C) Sis1 protein expression levels in yeast strains 74D-694 (74D) and W303 (W303). Cell lysates from wild-type or sis1- $\Delta$ cells expressing Sis1-21 from a plasmid were subjected to SDS-PAGE followed by immunoblotting with a Sis1-specific antibody. Antibody specific for $\mathrm{Ssc} 1$ was used as a loading control.

We reasoned that the observed differences in $\left[R N Q^{+}\right]$maintenance between these yeast strain backgrounds could be due either to differences in levels of expression of Sis1-121, or in some other characteristic that affected the requirement for chaperone activity for prion maintenance. To determine whether protein expression level might account for the observed differences in $\left[R N Q^{+}\right] /$ Sis1 requirements, we utilized a $\left[R N Q^{+}\right] 74 \mathrm{D}-694$ tester strain having a deletion of SIS 1 but expressing Sis1 from a URA3-marked plasmid, driven from its own promoter $\left(\left[R N Q^{+}\right]\right.$sis $1-\Delta[$ [SIS1-Sis1, URA3]). This strain was used to 'shuffle in' other SIS1 expressing plasmids by transformation, followed by growth on 5-fluoro-orotic acid (5-FOA), which counter-selects against plasmids carrying the URA3 allele. ${ }^{23}$ In this way, the effect of various SIS1 constructs was tested for maintenance of $\left[R N Q^{+}\right]$.

As a control, we first tested a single-copy plasmid containing the first 121 codons of Sis1 encoding the J-domain and G/F region under control of the SIS1 promoter (CEN SIS1-Sis1-121). ${ }^{24}$ Transformants that grew on 5-FOA were passaged on selective media twice and then the presence of the prion assayed by resolving prionspecific detergent-resistant aggregates using semi-

yeast prion in vivo can depend upon multiple factors. Prions can assume multiple conformations resulting in prion "variants" (also called strains) which exhibit differing heritable traits., ${ }^{5} 19-22$ Prion variants often differ in phenotypic strength and are therefore referred to as "strong" or "weak". For $\left[P S I^{+}\right]$, phenotypic strength also correlates with mitotic stability such that strong $\left[\mathrm{PSI}^{+}\right]$variants are more stable than weak variants. ${ }^{19-21}$ Additionally, extragenic polymorphisms inherent to a particular yeast strain can potentially alter prion behavior in vivo. The extent to which prion-chaperone interactions are affected by either yeast strain variation or prion variant differences is of particular interest, not only because the use of different strain backgrounds in various investigations makes general conclusions difficult to make, but because differences in behavior dependent on yeast strain background may provide clues to understand the basic mechanisms of prion behavior. Here we explore this idea with a focus on Sis1, concentrating on two issues, the reported difference in requirement for Sis1 domains in the maintenance of $\left[R N Q^{+}\right]^{10}$ and the affects of yeast strain background on the stability of $\left[\mathrm{PSI}^{+}\right]$ variants.

\section{Results}

Sis1 requirements by $\left[R N Q^{+}\right]$are consistent between yeast strains. Our laboratory's earlier investigation utilizing two distinct yeast strain backgrounds, W303 and 74D-694, found that Sis1's C-terminal peptide domain was required by $\left[R N Q^{+}\right]$only in 74D-694. ${ }^{10}$ However, in W303, $\left[R N Q^{+}\right]$was stable when only the J-domain and G/F-region were expressed (Sis1-121, Fig. 1A). ${ }^{10}$ denaturing detergent agarose gel electrophoresis (SDDAGE). ${ }^{25}$ As expected from previous results in reference $10,\left[R N Q^{+}\right]$was lost from the cell population as only soluble Rnq1 monomer was detectable on the gel (Fig. 1B). However, when Sis1-121 was expressed from a multi-copy plasmid $(2 \mu$ SIS1-Sis1-121), $\left[R N Q^{+}\right]$was maintained, indicating that, even in $74 \mathrm{D}-694$ cells, the J-domain and G/F region of Sis1 is sufficient for maintenance of $\left[R N Q^{+}\right]$.

Although the ability of Sis1-121 expressed from a high copy plasmid establishes the sufficiency of this fragment for maintenance of $\left[R N Q^{+}\right]$, this finding raises the possibly that higher levels of Sis1-121 protein are required for maintenance in 74D694, than in W303. To test the validity of this idea, or alternatively, establish whether Sis1-121 is expressed at lower levels in that strain background, we compared the abundance of Sis1-121 in extracts from 74D-694 and W303 cells by SDS-PAGE and immunoblotting with Sis1-specific antibodies. Indeed, W303 cells bearing CEN SIS1-Sis1-121 expressed approximately 2-fold more protein than 74D-694 cells (Fig. 1C). Expression from the multi-copy $2 \mu$ plasmid increased Sis1-121 expression in 74D694 by approximately $50 \%$ (Fig. 1C). To determine whether this difference in protein expression is specific to the Sis1-121 protein, we also examined the abundance of endogenous Sis1 in both strains. Full-length Sis1 was approximately $30 \%$ less abundant in the 74D-694 strain, indicating that there is a general strainspecific difference in Sis1 protein abundance between these two strains.

As a final consideration, we examined whether expression of Sis1- $\Delta \mathrm{G} / \mathrm{F}$, which has never been found to support $\left[R N Q^{+}\right]$ 
propagation, ${ }^{10,26}$ could in fact support $\left[R N Q^{+}\right]$if expressed from a multi-copy plasmid in the 74D-694 strain background. $\left[R N Q^{+}\right]$was lost from the cell population (Fig. 1B, center lane), reaffirming that the G/F region of Sis1 is essential for $\left[R N Q^{+}\right]$maintenance, regardless of yeast strain background. Thus, these results suggest that despite strain specific differences in Sis1 expression levels, the specific domain requirements of Sis1 by $\left[R N Q^{+}\right]$are likely to be uniform and independent of yeast strain background.

Similar kinetics of the loss of strong $\left[\mathrm{PSI}^{+}\right]$variants in two yeast strain backgrounds. We next turned our attention to the requirement for Sisl by the prion $\left[\mathrm{PSI}^{+}\right]$. In a report of our initial investigation, we speculated that the slow curing of $\left[\mathrm{PSI}^{+}\right]$by Sis1 repression was indicative of an alternative interaction scheme between $\left[\mathrm{PSI}^{+}\right]$, compared to $\left[R N Q^{+}\right]$, and the chaperone machinery. ${ }^{14}$ However, since only one variant of $\left[\mathrm{PSI}^{+}\right]$, a strong variant referred to hereafter as $\left[\mathrm{PSI}^{+}\right]^{\mathrm{STR}}$, and only one yeast strain background, W303, were examined quantitatively in our initial investigation, the question remained as to whether the unusual slow curing of $\left[\mathrm{PSI}^{+}\right]$upon Sis1 repression is a property of $\left[\mathrm{PSI}^{+}\right]$in general, or whether it is specific to a particular prion variant or yeast strain. For example, another Ssa co-chaperone, the nucleotide exchange factor Sse1, is required for the propagation of weak but not strong variants of $\left[\mathrm{PSI}^{+}\right] .{ }^{27,28}$ To begin to address this issue we repeated our initial Sis1 repression experiments in the W303 yeast strain background, but using a different strong $\left[\mathrm{PSI}^{+}\right]$variant, $\left[\mathrm{PSI}^{+}\right]^{\mathrm{SC} 4}$, distinct in origin from $\left[\mathrm{PSI}^{+}\right]^{\mathrm{STR} 21}\left[\mathrm{PSI}^{+}\right]^{\mathrm{SC} 4}$ is one of the best-characterized strong $\left[\mathrm{PSI}^{+}\right]$variants. $\left[\mathrm{PSI}^{+}\right]^{\mathrm{SC} 4}$ was created by polymerization of Sup35 in vitro, followed by transformation of [psi] yeast with the resulting amyloid material, which then served to seed prion formation in vivo. ${ }^{20,21,29}$

As in the past, we utilized a system having SIS1 under the control of the tet ${ }^{R}$ promoter (TETr), allowing repression of Sis1 synthesis to a minimal level sufficient for robust cell growth, upon addition of doxycycline..$^{14,16,18}$ Aliquots of the culture were removed and plated to observe colony color, as the presence or absence of $\left[\mathrm{PSI}^{+}\right]$can be easily assessed in these yeast strains due to $\left[P S I^{+}\right]$-dependent read-through of a stop codon in a gene required for adenine biosynthesis. [psi] cells form red colonies as a result of build up of a metabolic intermediate of the adenine biosynthetic pathway, whereas $\left[\mathrm{PSI}^{+}\right]$cells appear white or pink (Fig. 2A). ${ }^{30}$

Sis1 repression severely affected $\left[\mathrm{PSI}^{+}\right]^{\mathrm{SC} 4}$ propagation (Fig. 2A and B). By 35 generations more than half of the colonies were [ $p s i]$; by 79 generations, no pink colonies were observed, indicating the loss of the prion from the population. The loss of $\left[\mathrm{PSI}^{+}\right]^{\mathrm{SC} 4}$ was also verified using a biochemical assay, SDDAGE (Fig. 2C). The kinetics of $\left[\mathrm{PSI}^{+}\right]^{\mathrm{SC} 4}$ loss were similar but not identical to those observed previously for $\left[\mathrm{PSI}^{+}\right]^{\text {STR }}{ }^{14}{ }^{4} \mathrm{To}$ determine if the observed differences are properties of the prion variant, we again conducted Sis1 repression experiments with $\left[\mathrm{PSI}^{+}\right]^{\mathrm{STR}}$ and a third strong $\left[\mathrm{PSI}^{+}\right]$variant, $\left[\mathrm{PSI}^{+}\right]^{\mathrm{VH}} .{ }^{19}$ The curing curves for $\left[\mathrm{PSI}^{+}\right]^{\mathrm{STR}}$ and $\left[\mathrm{PSI}^{+}\right]^{\mathrm{VH}}$ were indistinguishable from $\left[\mathrm{PSI}^{+}\right]^{\mathrm{SC} 4}$ (Fig. 2D and E), demonstrating unambiguously that

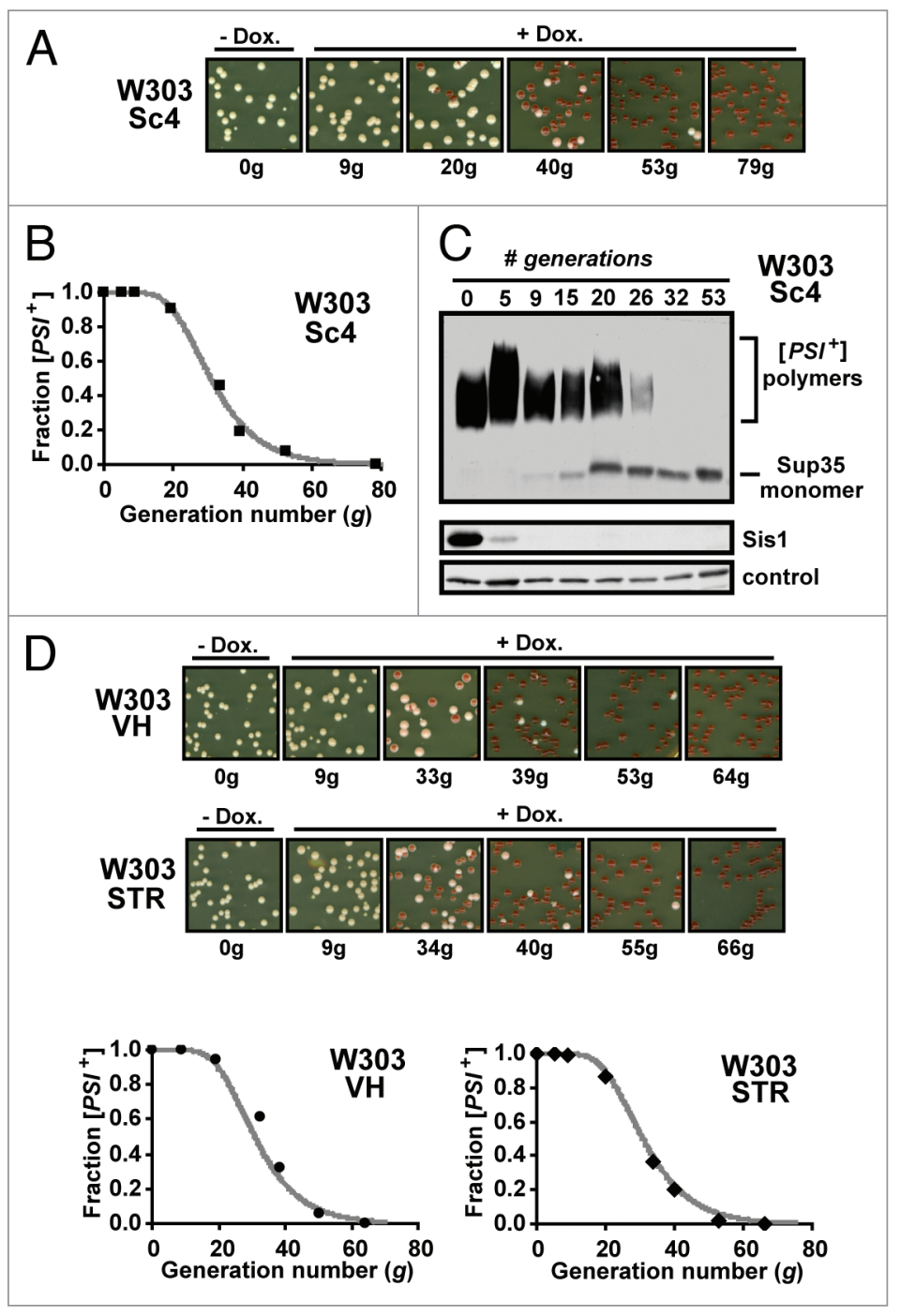

Figure 2. Loss of three distinct strong $\left[\mathrm{PSI}^{+}\right]$variants in the W303 genetic background upon Sis1 repression. (A and B) Time course of SIS1-repression of $\left[\mathrm{PSI}^{+}\right]^{\mathrm{SC} 4}$ cells. $\left[\mathrm{PSI} \mathrm{I}^{\mathrm{SC}}\right]^{\mathrm{SC}}$ cells were harvested after the indicated number of generations of growth in the presence of doxycycline and plated onto rich media. The fraction of cells remaining $\left[\mathrm{PSI}^{+}\right]^{\mathrm{SC} 4}$ (pink) vs. [psi] (red) was (A) determined and (B) plotted $(\mathrm{SC} 4, \mathbf{\square})$. The solid grey line represents a best-fit line through the data. (C) Lysates from sis1- $\Delta[\text { TETrSIS1], [PSI+ }]^{\mathrm{SC} 4}$ cells at various generations after addition of doxycycline, and from [psi] control cells, were resolved by SDDAGE (top part) and SDS-PAGE (bottom parts). Sup35 and Sis1 were visualized by immunoblotting with Sup35- and Sis1-specific antibodies. Antibody specific for Ssc1 was used as a loading control. (D and E) Time courses of SIS1-repression in cells bearing strong $\left[P S I^{+}\right]$variants $\left.\left[P S I^{+}\right]\right]^{\mathrm{VH}}(\mathrm{VH}, \bullet)$ and $\left[P S I^{+}\right]^{\text {STR }}(\mathrm{STR}, \bullet)$. Time courses were conducted as described in (A and $\mathrm{B}$ ). Grey line is the fit curve from (B) shown for comparison.

the slow curing of $\left[\mathrm{PSI}^{+}\right]$upon Sis1 repression is not a property of just a single strong $\left[\mathrm{PSI}^{+}\right]$variant, but instead appears to be a fundamental characteristic of multiple strong variants.

The experiments described above were all conducted in the W303 genetic background. However, the 74D-694 strain background has been very commonly used for the study of $\left[\mathrm{PSI}^{+}\right]$. To determine if the slow loss of strong $\left[\mathrm{PSI}^{+}\right]$upon Sis1 repression is caused by a particular aspect of the W303 proteome, we constructed 74D-694 Sis1 repression strains maintaining two 


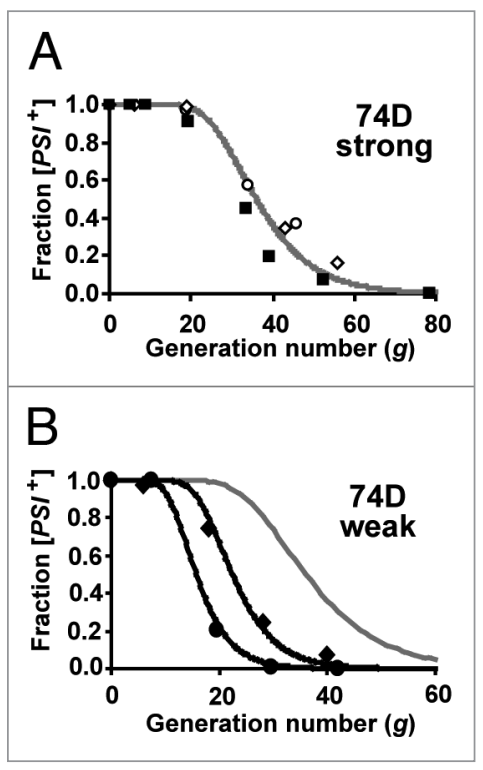

Figure 3. Kinetics of loss of strong and weak $\left[\mathrm{PSI}{ }^{+}\right]$variants in the $74 \mathrm{D}$ 694 genetic background upon Sis1 repression. (A) Time course of SIS1repression of strong variant-bearing cells. 74D-694 cells bearing strong $\left[P S I^{+}\right]$variants $\left[\mathrm{PSI}^{+}\right]^{\mathrm{SC} 4}(O)$ and $\left[\mathrm{PSI} \mathrm{I}^{+}\right]^{\mathrm{TRR}}(\diamond)$ were harvested after the indicated number of generations of growth in the presence of doxycycline and plated onto rich media. The fraction of cells remaining $\left[\mathrm{PSI}^{+}\right]$ based on colony color was plotted. For comparison, data for $\left[\mathrm{PS}^{+}\right]^{\mathrm{SC4}}$ in the W303 strain background, which also appears in Figure 2 is shown (घ). The solid grey line represents a best fit line through the data. (B) Weak $\left[P S I^{+}\right]$variants $\left[\mathrm{PSI}^{+}\right]^{\mathrm{SC} 37}(\bullet)$ and $\left[P S I^{+}\right]^{\mathrm{VL}}(\bullet)$, as in $(\mathrm{A})$. The grey line is the best fit line for strong strains from (A) shown for comparison (note: change in scale of the $x$-axis).

of the strong variants discussed above, $\left[P S I^{+}\right]^{\mathrm{STR}}$ and $\left[P S I^{+}\right]^{\mathrm{SC} 4}$. When subjected to Sis1 repression, both prions were lost with kinetics similar to those observed in the W303 background (Fig. 3A). This similarity of the kinetics of $\left[\mathrm{PSI}^{+}\right]$curing upon Sis1 repression strongly suggests that the slow curing relative to that observed for other prions studied thus far is a property of $\left[\mathrm{PSI}^{+}\right]$itself.

Rapid loss of weak $\left[\mathrm{PSI}^{+}\right]$variants in the $74 \mathrm{D}$ genetic background. The experiments described above were carried out using strong $\left[P S I^{+}\right]$variants. Weak $\left[P S I^{+}\right]$variants generally have fewer heritable seeds per cell than strong $\left[\mathrm{PSI}^{+}\right]$variants, creating a greater chance of prion loss during mitosis. ${ }^{5,21}$ Since Sis1 is believed to create heritable seeds via fragmentation, we predicted that weak $\left[\mathrm{PSI}^{+}\right]$variants would be cured more quickly than strong $\left[P S I^{+}\right]$variants upon repression of Sis1. To test this prediction, we examined two weak $\left[\mathrm{PSI}^{+}\right]$variants (called $\left[\mathrm{PSI}^{+}\right]^{\mathrm{SC} 37}$ and $\left.\left[P S I^{+}\right]^{\mathrm{VL}}\right)$ in the $74 \mathrm{D}-694$ strain background.$^{19}$ Both variants were cured rapidly by comparison to the strong $\left[\mathrm{PSI}^{+}\right]$variants, being completely eliminated from the cell population 45 generations after doxycycline addition (Fig. 3B). This result is informative in two ways: (1) it demonstrates the absolute requirement for Sis1 by $\left[\mathrm{PSI}^{+}\right]$is not dependent upon the strength of the prion variant and (2) the rapid loss of weak $\left[\mathrm{PSI}^{+}\right]$variants relative to strong $\left[\mathrm{PSI}^{+}\right]$variants is consistent with the role of Sis 1 in the creation of new heritable prion seeds.
Unusual kinetics of loss of weak $\left[\mathrm{PSI}^{+}\right]$variants in the W303 genetic background. We also assessed the loss of weak [PSI+] variants in the W303 genetic background using the same Sis1 repression system. As seen from the results described above, prion loss typically results in sigmoidal kinetics: prions are retained at a high frequency until the average seed number per cell is reduced to a low level at which time cells begin to inherit only one or no seeds, which leads to rapid prion loss from the population. ${ }^{31-33}$ An initial experiment with the $\left[\mathrm{PSI}^{+}\right]^{\mathrm{SC} 37}$ variant yielded atypical kinetics, with a significant fraction of the population retaining $\left[\mathrm{PSI}^{+}\right]^{\mathrm{SC} 37}$ for $>100$ generations. Repetitions of the experiment revealed this phenomenon to be highly reproducible. Figure 4A-C shows the results of one such experiment. As in the 74D-694 strain background, W303 $\left[\mathrm{PSI}^{+}\right]^{\mathrm{SC} 37}$ cells exhibited an initial rapid decrease in the percentage of $\left[\mathrm{PSI}^{+}\right]$cells after Sis1 repression. However, loss then slows, with $\left[\mathrm{PSI}^{+}\right]$cells disappearing from the cell population at nearly a linear rate. Two other weak variants, $\left[P S I^{+}\right]^{\mathrm{VL}}$ and $\left[P S I^{+}\right]^{\mathrm{VK}}$, behaved similarly (Fig. 4D and $\mathrm{E})$.

That the kinetics of three independently derived weak $\left[P S I^{+}\right]$ variants behaved so similarly in W303 strongly suggests that they are the result of a characteristic of this yeast strain background. However, we also wanted to determine if the observed kinetics might also be due to the presence of a mixed prion population, including a "cryptic" Sisl-independent [PSI+] variant, which in W303, but not 74D-694, remains after Sis1-dependent $\left[\mathrm{PSI}^{+}\right]$variants are eliminated. We reasoned that, if such were the case, $\left[\mathrm{PSI}^{+}\right]$cells present at the latter end of a curing experiment would be enriched for Sis1-independent $\left[\mathrm{PSI}^{+}\right]$variants. To test this hypothesis, we isolated six $\left[\mathrm{PSI}^{+}\right]$colonies from a sample of cells collected after 63 generations of cell culture in the presence of doxycycline, and restarted the Sis1-repression experiment. Representative SDDAGE gels for two of these strains are shown in Figure 5B. As a control, we also simultaneously retested the initial cell population (Fig. 5A). The results from the three cultures were indistinguishable: $\left[\mathrm{PSI}^{+}\right]^{\mathrm{SC} 37}$ was slowly eliminated from the cell population, indicating that the behavior of weak $\left[\mathrm{PSI}^{+}\right]$in $\mathrm{W} 303$ is not due to the selection for a Sis 1 independent $\left[\mathrm{PSI}^{+}\right]$variant. These results also demonstrate the reproducibility of the kinetics of loss, even after a tandem series of repressions of Sis1. Thus, Sis1 repression is not causing a permanent change of either the prion itself or the yeast strain. Rather the results are consistent with there being a fundamental difference between the W303 and 74D-694 yeast strain backgrounds that affects the kinetics of loss of weak $\left[\mathrm{PSI}^{+}\right]$variants following the depletion of Sis1.

\section{Discussion}

The goal of the experiments described here was to gain a better understanding of the relationship between chaperone function and prion propagation with respect to two common variables: yeast genetic background and the inherent variation of prions themselves. While the results presented here are limited in scope and thus do not permit us to make sweeping generalizations, they do, however, allow us to offer some cautionary notes regarding 
the interpretation of results obtained in different genetic backgrounds, and to suggest that some such differences may offer opportunities to dissect the complexities of prion biology.

Effect of prion variant differences on chaperone requirements. Prion variant differences can, in some cases, affect the sensitivity of yeast prions to ectopic chaperone expression. For example, Sse1, an Hsp110-type nucleotide exchange factor, is required for the propagation of weak but not strong $\left[\mathrm{PSI}^{+}\right]$variants. ${ }^{27,28}$ However, taking into consideration the results reported here and previously published results, there are still no prion variant-specific exceptions to the requirement for the "core" set of prion-maintaining chaperones, Hsp104, Ssa or Sis1, for prion propagation. Indeed, all currently available data support the idea that all naturally occurring, amyloid-forming yeast prions require these chaperones for stable propagation in yeast.

When studying the variants of $\left[P S I^{+}\right]$we were somewhat surprised to find that all three of the strong $\left[\mathrm{PSI}^{+}\right]$variants analyzed were cured with indistinguishable kinetics upon Sis1 repression, while greater variability existed among weak $\left[\mathrm{PSI}^{+}\right]$variants. The three weak variants were lost in the 74D-694 yeast strain background with faster kinetics than the strong variants, but those rates were not identical, indicating that differences between prion variant behavior may be reduced as phenotypic and mitotic strength increase. Because prion variants are a direct consequence of alternate amyloid folds, similar curing curves could indicate similar amyloid structure among these three strong $\left[\mathrm{PSI}^{+}\right]$variants, despite their distinct origins. One potential implication of this result is that strong $\left[\mathrm{PSI}^{+}\right]$variants may have converged upon an 'optimum' structure for $\left[\mathrm{PSI}^{+}\right]$that results in optimal mitotic stability. The fundamental requirement of attaining such a structure is that it must optimally balance fiber stability, fiber extension rates and fiber frangibility such that yeast cells maintain the highest possible number of heritable prion seeds. However, it is not implausible that more than one amyloid fold might satisfy these requirements.

Genetic basis of prion/chaperone dynamics: the influence of yeast strain background. Our comparisons of the effect of yeast genetic strain background, both reported here and previously published in reference 10,14 and 16, indicate that most of the fundamental conclusions reached about the requirement for Hsp104, Ssal and Sis1 hold for the most generally used yeast strain backgrounds. However differences do exist, as illustrated by the differences in expression from the SIS1 promoter in the W303 and 74D-694 genetic backgrounds. In the case of Sis1-121, but not full-length protein, this approximately two-fold variation made the difference between maintenance and loss of $\left[R N Q^{+}\right]$. Despite these small variations, the profound effect on $\left[R N Q^{+}\right]$indicates that the level of Sis1-121 in these strains is near the tipping point for prion curing. Thus, rather than being due to a fundamental difference in the sequences required for Sis 1 to function in prion propagation in the two backgrounds, the drastic difference in stability in the two strain backgrounds is due to subtle differences in expression levels.

Our investigation also uncovered an unusual genetic interaction affecting the curing of weak $\left[\mathrm{PSI}^{+}\right]$variants in the W303 genetic background. Three independently obtained weak variants were cured with the expected kinetics in 74D-694, but in W303 these variants, although eventually lost after Sis1 repression, were cured with atypical kinetics. The similar behavior of all three variants that were independently obtained suggests that this behavior is due to a difference in strain background between W303 and 74D-694. We entertained the possibility that 


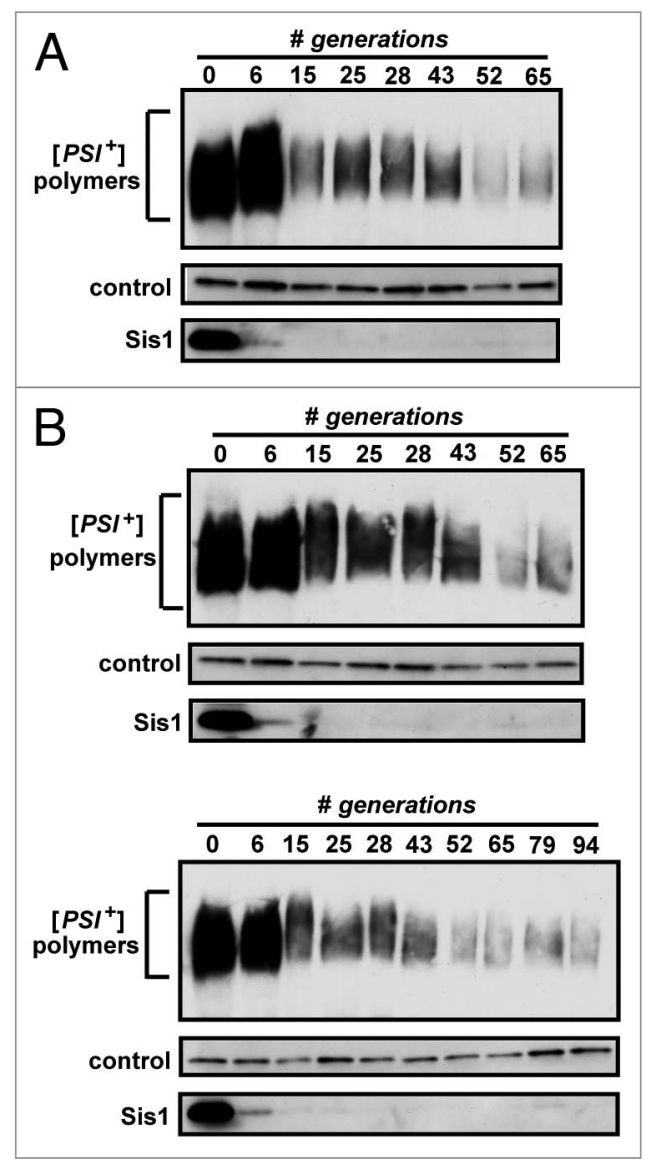

Figure 5. Unusual curing kinetics of weak $\left[P S I^{+}\right]$strains in $\mathrm{W} 303$ are not due to "cryptic" Sis1-independent $\left[\mathrm{PSI}^{+}\right]$variants. (A and B) SIS1 repression experiments described in the legend to Figure 4 were repeated using (A) the initial cell population (Fig. 4C, first lane) and (B) two individual $\left[\mathrm{PSI}^{+}\right]^{\mathrm{SC} 37}$ colonies isolated after 64 generations of Sis1 repression (Fig. 4C, last lane).

$\left[\mathrm{PSI}^{+}\right]^{\mathrm{SC} 37}$ and other weak $\left[\mathrm{PSI}^{+}\right]$variants might behave fundamentally differently in the W303 background to the extent that they are not truly "weak" variants. However, expected color phenotypes, sensitivity to Hsp104 overexpression and seed number (data not shown) all indicated that $\left[\mathrm{PSI}^{+}\right]^{\mathrm{SC} 37}$ behaves as a typical weak $\left[\mathrm{PSI}^{+}\right]$variant in W303, other than the kinetics of loss upon the repression of Sis1 synthesis. Thus, the observed differences between strain backgrounds are most likely to due to a factor, or set of factors, that are either constitutively expressed, or induced upon Sis1 repression, and that enhance the stability of weak but not strong $\left[\mathrm{PSI}^{+}\right]$in the absence of high Sis1 levels. We conducted a preliminary investigation testing likely candidates, including Hsp104, Ssa1-4, Ydj1 and Hsp26. However, expression of none were obviously different (data not shown). This trait may be polygenic, requiring a more thorough, global analysis to uncover.

In sum, the unusual kinetics of loss of weak $\left[\mathrm{PSI}^{+}\right]$variants is likely due to one or more polymorphisms in the W303 genetic background compared to 74D-694. Examining strain specific variation such as this, and others identified in the future, may provide information useful for understanding the complexities of prion propagation, which requires exquisite balancing between the addition of monomers to prion fibrils and their fragmentation. Analysis of complex traits amongst diverse genetic backgrounds has proven productive in other cases, such as ethanol tolerance, ${ }^{34}$ and may provide insight in prion biology as well.

\section{Materials and Methods}

Yeast strains and plasmids. Saccharomyces cerevisiae W303 and 74D-694 derived haploid strains were used throughout. W303 strains were derived from PJ513a: $\left[R N Q^{+}\right]\left[p s i^{i}\right]$ MAT a trp1-1 ura3-1 leu2-3,112 his3-11,15 ade2-1 can1-100 GAL2 met2-1 lys2-2. ${ }^{13}$ Strains having SIS1 under the control of the tetracycline repressible (TETr) promoter (sis1- $\Delta:: L E U 2$ [TETrSIS1]) were described previously in reference 14 and 18 . The 74D-694 strain used for plasmid shuffling was described in reference 16 . W303 $\left[\mathrm{PSI}^{+}\right]^{\mathrm{STR}}$, $\left[\mathrm{PSI}^{+}\right]^{\mathrm{SC} 4}$ and $\left[\mathrm{PSI}^{+}\right]^{\mathrm{SC} 37}$ strains and $74 \mathrm{D}-694$ $\left[\mathrm{PSI}^{+}\right]^{\mathrm{SC} 4}$, and $\left[\mathrm{PSI}^{+}\right]^{\mathrm{SC} 37}$ strains having ade1-14 (UGA) were gifts from Jonathan Weissman. ${ }^{20,21,35} 5 \mathrm{~V}-\mathrm{H} 19$ strains bearing $\left[P S I^{+}\right]^{\mathrm{VH}}$, $\left[P S I^{+}\right]^{\mathrm{VL}}$ or $\left[\mathrm{PSI}^{+}\right]^{\mathrm{VK}}$ were gifts from Chih-yen King and were used only for lysate transformations. ${ }^{19} \mathrm{~W} 303$ strains bearing $\left[P S I^{+}\right]^{\mathrm{VH}}$, $\left[P S I^{+}\right]^{\mathrm{VL}}$ or $\left[P S I^{+}\right]^{\mathrm{VK}}$, as well as $74 \mathrm{D}-694$ strains bearing $\left[P S I^{+}\right]$ STR or $\left[P S I^{+}\right]^{\mathrm{VL}}$ were constructed by yeast lysate transformation in which recipient $[p s i]$ spheroplasts were co-transformed with cell extracts of donor prion strains and a URA3-containing plasmid. ${ }^{36}$ Transformants were selected on medium lacking uracil, and patched onto rich media to analyze prion status based on colony color. The prion status of each strain was verified by prion curing on $\mathrm{GdnHCl}$ media and SDDAGE analysis. For use as controls $\left[\mathrm{rnq}^{-}\right]$and $\left[\mathrm{psi}^{-}\right]$strains were obtained by growth of the appropriate strain on liquid media containing $3 \mathrm{mM} \mathrm{GdnHCl}$ for two days.

The plasmid bearing TETrSIS1 and the URA3-bearing plasmid YCP50-SIS1-SIS1 used for plasmid shuffling experiments were described previously in reference 18 and 24 . Unless otherwise indicated, all others used in this study were based on the pRS plasmid series. ${ }^{37}$ Plasmid pRS314-SIS1-sis1-121 was from Yan and Craig, 2001. ${ }^{24}$ Plasmids pRS424-SIS1-sis1-121 and pRS424-SIS1-sis1 $\Delta \mathrm{G} / \mathrm{F}$ were constructed using standard molecular biology techniques.

Assays for prion loss. Time course experiments for $\left[\mathrm{PSI}^{+}\right]$ curing were executed as previously reported in reference 14 . Cell cultures were maintained in exponential growth phase by continual subculturing in YEPD in the presence of either $5 \mu \mathrm{g} / \mathrm{ml}$ doxycycline (Sigma) or $4 \mathrm{mM} \mathrm{GdnHCl}$ when indicated. Sis1 depleted cells remained viable for the duration of the experiment with a typical growth rate of $2.0-2.5 \mathrm{hrs} /$ generation. The presence or absence of $\left[\mathrm{PSI}^{+}\right]$was confirmed by observation of colony color on rich media where $\left[\mathrm{PSI}^{+}\right]$-mediated aggregation of Sup35, a translation termination factor, causes read-through of the premature nonsense codon in the ade1-14 mutant allele. ${ }^{38,39}$ Strains which are otherwise wild-type for adenine production appear pink or white in the presence of $\left[\mathrm{PSI}^{+}\right]$or dark red in the absence of $\left[\mathrm{PSI}^{+}\right]$due to the accumulation of a red intermediate when adenine production is blocked. ${ }^{40}$ For the purposes of kinetic analyses, the curing of $\left[\mathrm{PSI}^{+}\right]$was monitored by colony 
color counting assays ${ }^{41}$ with sectored colonies being counted as $\left[\mathrm{PSI}^{+}\right] .{ }^{31}$ Kinetic models used to fit prion curing data were described in reference 14 . Semi-denaturing detergent agarose gel electrophoresis (SDDAGE) ${ }^{25}$ was used to verify presence or loss of prions by resolving detergent resistant aggregates and was performed as described in reference 14 .

SDS-PAGE and immunoblot analysis. Total protein extracts were prepared by harvesting yeast cells in mid-log phase followed by lysis in $\mathrm{NaOH}$. The resulting protein extracts were analyzed by SDS-PAGE and immunoblot analysis. Antibodies specific to Sis1, Sup35 and Rnq1 have been described in reference 14 and 18. Densitometry measurements used to estimate relative protein expression levels were made using the program ImageJ. ${ }^{42}$

\section{References}

1. Chiti F, Dobson CM. Protein misfolding, functional amyloid and human disease. Annu Rev Biochem 2006; 75:333-66.

2. Sondheimer N, Lindquist S. Rnq1: an epigenetic modifier of protein function in yeast. Mol Cell 2000; 5:163-72.

3. Derkatch IL, Bradley ME, Hong JY, Liebman SW. Prions affect the appearance of other prions: the story of [PIN(+)]. Cell 2001; 106:171-82.

4. Wickner RB, Masison DC, Edskes HK. [PSI] and [URE3] as yeast prions. Yeast 1995; 11:1671-85.

5. Derkatch IL, Chernoff YO, Kushnirov VV, IngeVechtomov SG, Liebman SW. Genesis and variability of [PSI] prion factors in Saccharomyces cerevisiae. Genetics 1996; 144:1375-86.

6. Crow ET, Li L. Newly identified prions in budding yeast, and their possible functions. Semin Cell Dev Biol $2011 ; 22: 452-9$

7. Tuite MF, Koloteva-Levin N. Propagating prions in fungi and mammals. Mol Cell 2004; 14:541-52.

8. Chernoff YO, Lindquist SL, Ono B, Inge-Vechtomov SG, Liebman SW. Role of the chaperone protein Hsp104 in propagation of the yeast prion-like factor [psi ${ }^{+}$. Science 1995; 268:880-4.

9. Song Y, Wu YX, Jung G, Tutar Y, Eisenberg E, Greene LE, et al. Role for Hsp70 chaperone in Saccharomyces cerevisiae prion seed replication. Eukaryot Cell 2005; 4:289-97.

10. Sondheimer N, Lopez N, Craig EA, Lindquist $S$. The role of Sis 1 in the maintenance of the $\left[\mathrm{RNQ}^{+}\right]$prion. EMBO J 2001; 20:2435-42.

11. Masison DC, Kirkland PA, Sharma D. Influence of Hsp70s and their regulators on yeast prion propagation. Prion 2009; 3:65-73.

12. Kampinga $\mathrm{HH}$, Craig EA. The HSP70 chaperone machinery: J proteins as drivers of functional specificity. Nat Rev Mol Cell Biol 2010; 11:579-92.

13. Sahi C, Craig EA. Network of general and specialty J protein chaperones of the yeast cytosol. Proc Natl Acad Sci USA 2007; 104:7163-8.

14. Higurashi T, Hines JK, Sahi C, Aron R, Craig EA. Specificity of the J-protein Sis 1 in the propagation of 3 yeast prions. Proc Natl Acad Sci USA 2008; 105:16596-601.

15. Tipton KA, Verges KJ, Weissman JS. In vivo monitoring of the prion replication cycle reveals a critical role for Sis1 in delivering substrates to Hsp104. Mol Cell 2008; 32:584-91.

\section{Disclosure of Potential Conflicts of Interest}

No potential conflicts of interest were disclosed.

\section{Acknowledgments}

We thank Mick Tuite for Sup35 antibodies; Jonathan Weissman and Chih-yen King for strong and weak $\left[\mathrm{PSI}^{+}\right]$strains; members of the Craig laboratory for thoughtful comments on the manuscript. This work was supported by the National Institute of Health Grant GM31107 (E.A.C.) and a National Institute of Health NRSA postdoctoral fellowship GM846542 (J.K.H.) www.nih.gov.
16. Hines JK, Li X, Du Z, Higurashi T, Li L, Craig EA. [SWI], the prion formed by the chromatin remodel ing factor Swil, is highly sensitive to alterations in Hsp70 chaperone system activity. PLoS Genet 2011; 7:1001309.

17. Kirkland PA, Reidy M, Masison DC. Functions of yeast Hsp40 chaperone Sis1p dispensable for prion propagation but important for prion curing and protection from prion toxicity. Genetics 2011; 188:565-77.

18. Aron R, Higurashi T, Sahi C, Craig EA. J-protein co-chaperone Sis 1 required for generation of $\left[\mathrm{RNQ}^{+}\right]$ seeds necessary for prion propagation. EMBO J 2007; 26:3794-803.

19. King CY. Supporting the structural basis of prion strains: induction and identification of [PSI] variants. J Mol Biol 2001; 307:1247-60.

20. Tanaka M, Chien P, Naber N, Cooke R, Weissman JS. Conformational variations in an infectious protein determine prion strain differences. Nature 2004; 428:323-8.

21. Tanaka M, Collins SR, Toyama BH, Weissman JS. The physical basis of how prion conformations determine strain phenotypes. Nature 2006; 442:585-9.

22. Bradley ME, Edskes HK, Hong JY, Wickner RB, Liebman SW. Interactions among prions and prion "strains" in yeast. Proc Natl Acad Sci USA 2002; 99:16392-9.

23. Sikorski RS, Boeke JD. In vitro mutagenesis and plasmid shuffling: from cloned gene to mutant yeast. Methods Enzymol 1991; 194:302-18.

24. Yan W, Craig EA. The glycine-phenylalanine-rich region determines the specificity of the yeast Hsp40 Sis1. Mol Cell Biol 1999; 19:7751-8.

25. Kryndushkin DS, Alexandrov IM, Ter-Avanesyan MD, Kushnirov VV. Yeast $\left[\mathrm{PSI}^{+}\right]$prion aggregates are formed by small Sup 35 polymers fragmented by Hsp104. J Biol Chem 2003; 278:49636-43.

26. Lopez N, Aron R, Craig EA. Specificity of class II Hsp40 Sis1 in maintenance of yeast prion $\left[\mathrm{RNQ}^{+}\right]$. Mol Biol Cell 2003; 14:1172-81.

27. Fan Q, Park KW, Du Z, Morano KA, Li L. The role of Sse1 in the de novo formation and variant determination of the $\left[\mathrm{PSI}^{+}\right]$prion. Genetics 2007; 177:1583-93.

28. Kryndushkin D, Wickner RB. Nucleotide exchange factors for $\mathrm{Hsp} 70$ s are required for [URE3] prion propagation in Saccharomyces cerevisiae. Mol Biol Cell 2007; 18:2149-54.
29. Tanaka M, Weissman JS. An efficient protein transformation protocol for introducing prions into yeast. Methods Enzymol 2006; 412:185-200.

30. Wickner RB, Edskes HK, Shewmaker F, Nakayashiki T. Prions of fungi: inherited structures and biological roles. Nat Rev Microbiol 2007; 5:611-8.

31. Cox B, Ness F, Tuite M. Analysis of the generation and segregation of propagons: entities that propagate the [PSI ${ }^{+}$] prion in yeast. Genetics 2003; 165:23-33.

32. Byrne LJ, Cox BS, Cole DJ, Ridout MS, Morgan BJ, Tuite MF. Cell division is essential for elimination of the yeast $\left[\mathrm{PSI}^{+}\right]$prion by guanidine hydrochloride. Proc Natl Acad Sci USA 2007; 104:11688-93.

33. Ness F, Ferreira P, Cox BS, Tuite MF. Guanidine hydrochloride inhibits the generation of prion "seeds" but not prion protein aggregation in yeast. Mol Cell Biol 2002; 22:5593-605.

34. Lewis JA, Elkon IM, McGee MA, Higbee AJ, Gasch AP. Exploiting natural variation in Saccharomyces cerevisiae to identify genes for increased ethanol resistance. Genetics 2010; 186:1197-205.

35. Osherovich LZ, Weissman JS. Multiple Gln/Asn-rich prion domains confer susceptibility to induction of the yeast [PSI(+)] prion. Cell 2001; 106:183-94.

36. Brachmann A, Baxa U, Wickner RB. Prion generation in vitro: amyloid of Ure2p is infectious. EMBO J 2005; 24:3082-92.

37. Mumberg D, Muller R, Funk M. Yeast vectors for the controlled expression of heterologous proteins in different genetic backgrounds. Gene 1995; 156:119-22.

38. Inge-Vechtomov SG, Tikhodeev ON, Karpova TS Selective systems for obtaining recessive ribosomal suppressors in saccharomycete yeasts. Genetika 1988; 24:1159-65.

39. Cox BS. [PSI], a cytoplasmic suppressor of supersuppression in yeast. Heredity 1965; 20:505-21.

40. Bousset L, Savistchenko J, Melki R. Assembly of the asparagine- and glutamine-rich yeast prions into protein fibrils. Curr Alzheimer Res 2008; 5:251-9.

41. Eaglestone SS, Ruddock LW, Cox BS, Tuite MF. Guanidine hydrochloride blocks a critical step in the propagation of the prion-like determinant $[\mathrm{PSI}(+)]$ of Saccharomyces cerevisiae. Proc Natl Acad Sci USA 2000; 97:240-4.

42. Abramoff MD, Magelhaes PJ, Ram SJ. Image Processing with ImageJ. Biophotonics International 2004; 11:36-42. 